\title{
Jean-Pierre Deffontaines, agronome, géographe, humaniste
}

Jean-Pierre Deffontaines nous a quittés le 25 octobre dernier. Le comité de rédaction lui consacre cette rubrique sous trois formes : un hommage de notre collectif au compagnon de route et à l'ami, une illustration de sa créativité artistique indissociable de son approche scientifique, un texte, rédigé en collaboration, sur sa prise en compte du sensible dans son approche des paysages.

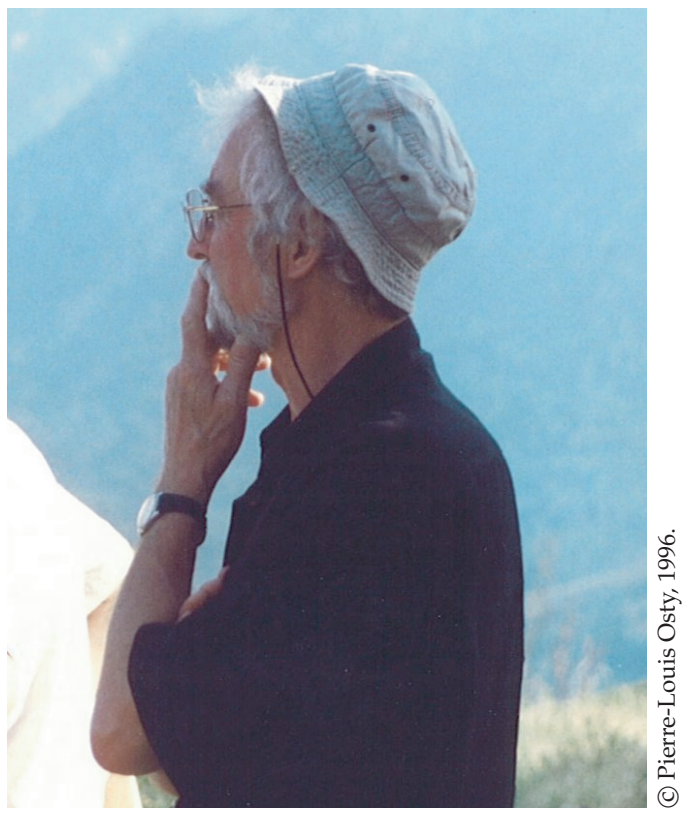

Avec Jean-Pierre Deffontaines, c'est une des personnalités de la revue Natures Sciences Sociétés qui disparaît. $C^{\prime}$ est aussi un ami, dont la sensibilité se manifestait dans les relations qu'il avait avec les autres, toutes empreintes de délicatesse, de cordialité, d'empathie. Pour beaucoup d'entre nous, c'est aussi l'affection qui est en deuil.

Il a fait partie dès le début, c'est-à-dire dès le milieu des années 1980, de l'aventure qui a conduit à la création de la revue en 1993. Ces vingt années de fidélité à ce projet et d'énergie combative mise à son service témoignent de sa constance dans ses engagements pour défendre les conceptions de la recherche qui lui tenaient à cœur. Assidu aux réunions du comité de rédaction, il en était un élément moteur par ses interventions dans les discussions et par les initiatives régulières qu'il prenait pour alimenter la revue de ses idées. Il apportait dans le travail du collectif une contribution irremplaçable en raison de l'originalité et de la finesse de son point de vue, produit d'un croisement - qui lui appartient en propre entre l'agronomie et la géographie, dont il fit bénéficier de nombreux jeunes collègues qu'il a pris soin d'accompagner dans leurs débuts professionnels.

Géographe, il l'était par son père, Pierre, qui dirigea la collection «Géographie humaine » de la NRF (Gallimard) - collection interdisciplinaire, puisqu'elle était ouverte aux anthropologues et aux historiens, et délibérément orientée vers une géographie des relations entre humains et milieux. Agronome, il l'était par ses maîtres, René Dumont et Stéphane Hénin. Sur les conseils du premier, qu'il était allé voir au retour de son service militaire pour lui proposer ses services, il a rencontré le second, qui l'a pris comme assistant en 1961. Tout au long de sa carrière, chemin faisant, comme il aimait à dire, en citant Antonio Machado, il s'est consacré à une fécondation croisée des deux disciplines. Pour lui, à la géographie, l'agronomie apporte sa compréhension des faits techniques, tandis qu'à la seconde, la première offre son intelligence des organisations spatiales.

Sans doute a-t-il commencé par exercer son regard d'agronome en travaillant, au départ, avec «Monsieur Hénin », sur les «potentialités des terrains ». Alors que beaucoup parlaient encore de «vocation des sols », Hénin et Deffontaines préférèrent la notion de «potentialité en fonction des usages ». Mais le courant dominant de l'agronomie caractérise les potentialités et les usages à l'échelle de la parcelle, ce que Jean-Pierre Deffontaines ne tarde pas à critiquer. Dès son entrée à l'Inra, en 1965, comme chargé de recherche au Service d'expérimentation et d'information (SEI), il donne à l'agronomie une dimension spatiale. Il la développe au sein du groupe 
de recherches Inra-ENSSAA, qui publie en 1977 le fameux ouvrage Pays, paysans, paysages dans les Vosges $d u$ Sud. Dès cette époque, les auteurs identifient la demande croissante de préservation des ressources naturelles et de maintien de l'agriculture en montagne. Pour y répondre, ils étudient le rôle des pratiques agricoles et de leur diversité sur la transformation de l'espace. Les principaux concepts au cœur de la pensée scientifique de Jean-Pierre Deffontaines se mettent alors en place. Concepts empruntés : celui de pratiques aux ethnologues, ceux de paysages et de terroirs aux géographes. Empruntés, mais non volés. Empruntés et rendus enrichis par le regard de l'agronome et son intelligence des systèmes techniques. Car Jean-Pierre Deffontaines met l'analyse du fait technique au cœur de ses réflexions; il en fait la charnière entre les sciences de la nature et les sciences humaines.

Relié au terme de système agraire, celui de développement définit le nouveau département de recherche (le SAD), créé à l'Inra en 1979 par Jacques Poly et placé sous la houlette de Bertrand Vissac. Jean-Pierre Deffontaines participe activement à sa constitution. Il dirige une unité sur trois sites (Versailles, Dijon et Mirecourt); il y impulse des travaux pluridisciplinaires qui accordent une place importante à la diversité des types d'exploitation et à l'observation agronomique du paysage. Il fut l'un des initiateurs du programme Vittel, l'un des premiers projets de recherche interdisciplinaire, à la fin des années 1980, sur les relations entre agriculture et qualité des eaux, mené en collaboration entre plusieurs institutions de recherche et en partenariat étroit avec les acteurs impliqués localement. Le paysage est pour lui « une conséquence des pratiques qui en révèle aussi les causes, car il reflète les contraintes de milieu et de structures qui conditionnent leur mise en œuvre... [Aussi est-ce] un support d'information privilégié pour la connaissance des pratiques actuelles et passées et leur compréhension ».

Jean-Pierre Deffontaines a joué un rôle majeur dans la dynamique de recherche qui s'est développée autour du paysage à partir des années 1970. Par son ouverture d'esprit, il est apprécié dans les très nombreuses instances de réflexion et d'évaluation auxquelles il participe : le comité «Espace et cadre de vie» du ministère de l'Environnement, le comité «Land Use » de la $\mathrm{CE}$, la cellule « Paysage et aménagement rural » du ministère de l'Agriculture, les nombreux comités mis en place par la DGRST. Dans un contexte qui tendait à opposer des approches écologiques aux sciences de l'homme, il avait d'abord montré que des disciplines biotechniques comme l'agronomie pouvaient aider à établir des passerelles. Il avait également été un des premiers à souligner l'importance à donner aux paysages de l'ordinaire et aux représentations des habitants, ce qui a permis de prendre de la distance avec des conceptions qui privilégiaient l'exceptionnel et l'élitaire.

Il était très attaché au développement de collaborations avec les autres établissements de recherche ou d'enseignement. Il avait notamment apporté soutien et conseils pour le démarrage d'une activité de recherche au Cemagref et à l'École nationale supérieure du paysage de Versailles. Après avoir reçu le prix scientifique XavierBernard de l'Académie d'agriculture pour l'ensemble de ses travaux sur le paysage et le développement local, peu de temps avant sa retraite, il est admis définitivement comme membre titulaire de cette institution dans la section VII : « Ressources naturelles, aménagement de l'espace et environnement ».

On a du mal à imaginer que la retraite aurait pu arrêter ses activités. Il fait encore partie de différents comités scientifiques, comme celui du programme Politiques publiques et paysages, financé par le ministère de l'Écologie et du Développement durable de 1998 à 2004, ou celui des «Entretiens du Pradel», qui, depuis 2000, se tiennent tous les deux ans dans le domaine historique d'Olivier de Serres. Au sein de l'Académie d'agriculture de France, il joue un rôle moteur dans l'élaboration du "Graphe d'activité de la section VII », qui s'appuie sur le triptyque «Homme-Nature-Techniques » dont procède la création de la section. Juste avant son dernier été, si douloureux, sans le moindre répit, il donnait encore sa dernière «leçon de paysage » devant un public de lycéens, dans le style épuré de ceux qui comptent le temps qui reste.

On trahirait sa mémoire si l'on n'évoquait pas sa sensibilité personnelle, exceptionnelle, et toute esthétique, aux «formes » des paysages, la forme (et, par conséquent, la morphologie, et du coup l'observation visuelle) devenant pour lui un concept majeur de l'analyse. Elle se traduisait tout particulièrement par ses talents de dessinateur, de photographe et, plus originaux, de sculpteur sur bois. Donnant forme à des formes, d'un tronc d'arbre trouvé en forêt, il façonnait un paysage. Nous savons ce que cette sensibilité avait de profond chez lui. Source de création plastique, elle alimentait aussi sa posture scientifique. Celle-ci y trouvait son inspiration, son origine, et son originalité précisément. C'est notamment la confrontation avec architectes et plasticiens qui l'avait conduit à aborder la question des formes et de leur dynamique. À travers sa démarche, il nous donne ainsi une superbe illustration, et un superbe exemple, de ce qu'est une science «humaine", c'est-à-dire une science plongeant ses racines dans les ressorts profonds de ce qui fait l'humanité. 